\title{
Magnetic Resonance Imaging Characteristics of Tumefactive Demyelinating Lesions
}

\author{
Jiro Akimoto, Tomohiro Suda, Ryo Hashimoto, Hirokazu Fukuhara, Michihiro Kohno \\ Department of Neurosurgery, Tokyo Medical University, Tokyo, Japan \\ Email: jiroaki@gmail.com
}

Received 16 March 2015; accepted 3 May 2015; published 6 May 2015

Copyright (C) 2015 by authors and Scientific Research Publishing Inc.

This work is licensed under the Creative Commons Attribution International License (CC BY). http://creativecommons.org/licenses/by/4.0/

c) (i) Open Access

\section{Abstract}

Tumefactive demyelinating lesions (TDLs) are often misdiagnosed as brain tumors. To identify imaging characteristics of TDLs for their proper diagnosis, their differences from malignant gliomas, etc., were investigated based on 3-Tesla magnetic resonance imaging (MRI) and MR spectroscopy data that was obtained from 5 patients diagnosed with TDLs in our hospital. MRI displayed a paraventricular lesion with a maximum diameter of $\geq 2 \mathrm{~cm}$. Diffusion-weighted imaging (DWI) displayed a region of bright signal intensity at the margin of the lesion, and apparent diffusion coefficient (ADC) imaging displayed high signal intensity at the core of the lesion. The mass effect was mild relative to the size of the lesion, and the edema around the lesion was mild to moderate. Gadolinium-enhanced MRI displayed a characteristic open-ring sign, in which the ring of enhancement was incomplete (open towards the cortex), with the medullary vein running through the lesion, resulting in the enhancement of the surrounding area. MR spectroscopy displayed an increase in the choline peak, a decrease in the $\mathrm{N}$-acetyl aspartate (NAA) peak, the presence of lactate/lipid peaks, and the presence of $\beta, \gamma$-glutamate-glutamine (GLX) peaks that are slightly shifted to a higher resonance frequency $(2.1-2.4 \mathrm{ppm})$ from the NAA peak. Taken together, the following main characteristics were identified as imaging features of TDLs: bright rim on DWI, a high signal core on ADC imaging, an open-ring sign, and $\beta, \gamma$-GLX peaks on proton MR spectroscopy. We concluded that the key to the improvement of neurological symptoms of patients is the prompt initiation of steroid treatment following the accurate diagnosis of TDLs.

\section{Keywords}

Tumefactive Demyelinating Lesions, Open-Ring Sign, MRI, MR Spectroscopy

\section{Introduction}

Patients with neurological symptoms whose diagnosis and subsequent choice of treatment are difficult due to the

How to cite this paper: Akimoto, J., Suda, T., Hashimoto, R., Fukuhara, H. and Kohno, M. (2015) Magnetic Resonance Imaging Characteristics of Tumefactive Demyelinating Lesions. J. Biomedical Science and Engineering, 8, 321-326. 
absence of typical imaging features are sometimes suspected of having a brain tumor and are referred to neurosurgeons. In fact, pseudo-tumor lesions have occasionally been reported, such as cerebral infarction that may be mistaken for a tumor, inflammatory lesions including those caused by infections, congenital diseases including cortical dysplasia, and conditions caused by minor trauma. The differential diagnosis made by the attending physician is hence crucial for the accurate diagnosis of and to provide appropriate treatment to patients displaying atypical imaging features.

We recently encountered 5 patients who were referred to our hospital with a diagnosis of malignant glioma, but were diagnosed as having tumefactive demyelinating lesions (TDLs). In this study, we aimed to identify the imaging characteristics of TDLs, which are crucial for its accurate diagnosis, as well as for the differential diagnosis of pseudo-tumor diseases [1]-[4].

\section{Materials and Methods}

This study included 5 patients ( 3 men and 2 women), aged between 32 and 58 years (mean age: 48 years). Their initial symptoms were local brain symptoms that corresponded to the specific brain area of the lesion: 3 patients had hemisensory disturbance and paralysis, 1 patient had homonymous hemianopsia, and 1 patient had aphasia. None of the patients had symptoms of intracranial hypertension, such as headache or nausea (Table 1). In one of the patients, the symptoms were preceded by influenza virus infection. Four of the patients had a solitary lesion,

Table 1. A summary of the clinical features, spinal fluid data, and imaging findings of the 5 patients. F: female, M: male, CSF: cerebrospinal fluid, MBP: myelin basic protein, IgG: immunoglobulin G, DWI: diffusion-weighted image, ADC: apparent diffusion coefficient, Cho: choline, NAA: N-acetyl aspartate, Lac: lactate, Lip: lipid, GLX: glutamate and glutamine.

\begin{tabular}{|c|c|c|c|c|c|}
\hline & Case 1 & Case 2 & Csse 3 & Case 4 & Case 5 \\
\hline Age/gender & $48 / \mathrm{F}$ & $58 / \mathrm{M}$ & $32 / \mathrm{M}$ & $52 / \mathrm{F}$ & $42 / \mathrm{M}$ \\
\hline Symptoms & $\begin{array}{l}\text { Hemiparesis, } \\
\text { Heminumbness }\end{array}$ & Sensory aphasia & Heminumbness & Hemiparesis/Heminumbness & Hemianopsia \\
\hline \multicolumn{6}{|l|}{ CSF analysis } \\
\hline Protein (mg/dl) & 56 & 75 & 32 & 52 & 32 \\
\hline Glucose (mg/dl) & 67 & 48 & 60 & 63 & 69 \\
\hline Cell count $(/ \mu \mathrm{l})$ & 63 & 81 & 27 & 5 & 6 \\
\hline MBP (pg/ml) & Not examined & Not examined & $<40$ & $<40$ & $<40$ \\
\hline Oligoclonal IgG band & $(-)$ & $(-)$ & $(-)$ & $(-)$ & $(-)$ \\
\hline \multicolumn{6}{|l|}{ Lesion } \\
\hline Number & Single & Single & Multiple & Single & Single \\
\hline Location & Left parietal lobe & $\begin{array}{c}\text { Left } \\
\text { temporal lobe }\end{array}$ & $\begin{array}{c}\text { Bilateral } \\
\text { parietal lobe }\end{array}$ & $\begin{array}{c}\text { Right } \\
\text { corona radiata }\end{array}$ & $\begin{array}{l}\text { Right occipital } \\
\text { lobe }\end{array}$ \\
\hline \multicolumn{6}{|l|}{ MRI } \\
\hline Bright rim on DWI & $(+)$ & $(+)$ & $(+)$ & $(+)$ & $(+)$ \\
\hline $\begin{array}{l}\text { High signal } \\
\text { core on ADC }\end{array}$ & Not examined & Not examined & $(+)$ & Not examined & $(+)$ \\
\hline Open ring enhancement & $(+)$ & $(+)$ & $(-)$ & Closed ring & $(+)$ \\
\hline \multicolumn{6}{|l|}{ MRS } \\
\hline High Cho/NAA & Not examined & Not examined & $(+)$ & Not examined & $(+)$ \\
\hline Lac/Lip peak & Not examined & Not examined & $(+)$ & $(+)$ & $(+)$ \\
\hline$\beta, \gamma$-GLX peak & Not examined & Not examined & $(+)$ & $(+)$ & $(+)$ \\
\hline
\end{tabular}


and 1 patient had multifocal lesions. The lesions were located adjacent to the central sulcus in 3 patients, in the right occipital lobe in 1 patient, and in the left temporal lobe in 1 patient. Blood biochemistry data at the time of admission showed no noteworthy abnormal findings. Spinal fluid analysis revealed that the initial pressure of all patients was within the normal range, with clear, water-like fluid, and also showed slightly increased protein concentrations in 3 patients and the presence of several mononuclear cells in all the patients. None of the patients showed an increased myelin basic protein level in the spinal fluid nor the presence of oligoclonal immunoglobulin $\mathrm{G}$ bands.

The 3 patients with a solitary lesion underwent lesion removal with a small craniotomy (biopsy in 2 patients and total removal in 1 patient), and the other 2 patients were subjected to treatment without surgery based on diagnostic imaging. The bulk of the tissue removed was composed of densely accumulated macrophages engulfing myelin. In the surrounding area, prominent reactive astrocytosis and granular mitosis (Creutzfeldt cells) were observed, with T-cell-dominant lymphocyte infiltration in the perivascular area. Based onthese findings, the patients were diagnosed with inflammatory demyelination. All the patients subsequently received 3 cycles of pulse therapy with methylprednisolone, and the disappearance of the lesions was confirmed by imaging. Although symptom improvement was observed in all the patients, their mild neurological symptoms persisted.

Detailed imaging analysis using 3-Tesla magnetic resonance imaging (MRI) (Achieva ${ }^{\circledR}$; Phillips Inc.) was performed in the 5 patients. For basic imaging, T1-weighted imaging, T2-weighted imaging, fluid-attenuated inversion recovery (FLAIR), and diffusion-weighted imaging (DWI) were performed. In addition, apparent diffusion coefficient (ADC) imaging, T2-star imaging, and gadolinium-enhanced imaging were performed. Moreover, proton MR spectroscopy (MRS) was performed using point-resolved spectroscopy with a repetition time of $1200 \mathrm{msec}$ and an echo time of $288 \mathrm{msec}$. For a $10 \times 10 \mathrm{~cm}^{2}$ field-of-view, chemical shift images were obtained with single voxel and multiple voxel techniques, based on the spectral distribution of each metabolite. Using the imaging dataof the 5 patients, we attempted to identify characteristic features of TDLs on MRI and MRS.

\section{Results}

\subsection{MR Imaging}

In all the patients, a lesion with a maximum diameter of $\geq 2 \mathrm{~cm}$ displaying low signal intensity on T1W1 and high signal intensity on T2WI and FLAIR was observed. DWI displayed high or bright signal intensity in the surrounding area and low signal intensity at the core. ADC imaging displayed high signal intensity at the core. Contrast-enhanced MRI showed irregular contrast enhancement that appeared to delineate the border of the surrounding area of the lesion. The enhancement was intense in the deep white matter, with the ring of enhancement being open towards the surface of the brain, showing a so-called open-ring sign. T2-star imaging and contrast-enhanced imaging displayed vascular signals that appeared to involve a vein running through the core of the lesion, with the enhancement of the surrounding area. Although there was moderate white matter edema surrounding the lesion, the mass effect on the surrounding structure appeared to be mild relative to the size of the lesion (Figures 1(A)-(F), Figure 1(H) and Figure 1(I)).

\subsection{Proton MR Spectroscopy}

Proton MR spectroscopy was performed in 3 patients. In all patients, a slight increase in the choline (Cho) peak, a decrease in the N-acetylaspartate (NAA) peak, and the presence of lactate/lipid (Lac/Lip) peaks were observed at the core. In the surrounding area of the lesion, the decrease in the NAA peak was slight, and the Cho/NAA ratio was higher than that observed at the core. At the core and edge of the lesion, waveforms called $\beta, \gamma$-GLX (glutamate/glutamine) peaks that are slightly shifted to a higher resonance frequency (2.2 - $2.4 \mathrm{ppm}$ ) from the NAA peak, which are not observed in the normal brain, were identified (Figure 1(G) and Figure 1(I)).

\section{Discussion}

The recognition of the pathological features of TDLs has gradually increased among neurosurgeons, owing to the increase in the number of case reports. However, there are still many reports of patients with a preoperative diagnosis of malignant melanoma, who are pathologically diagnosed as having TDLs after total removal of the lesion by craniotomy. Taking into account that demyelination in a broad sense is an inflammatory disease based 


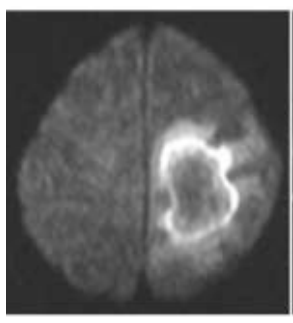

(a)

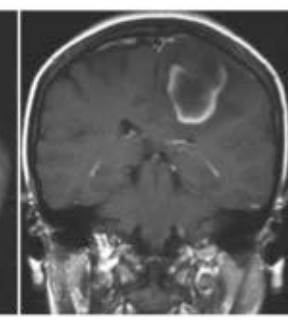

(b)

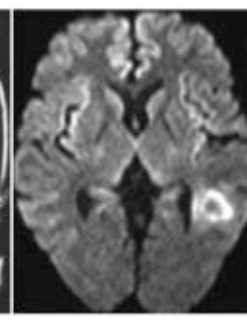

(c)

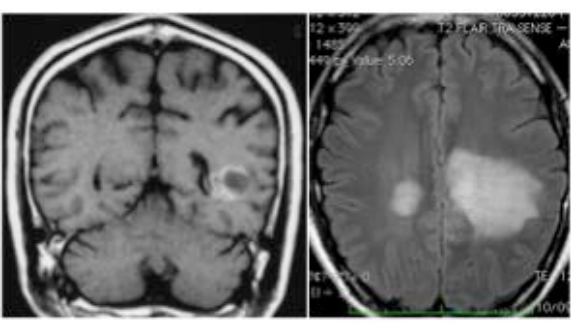

(d)

(e)

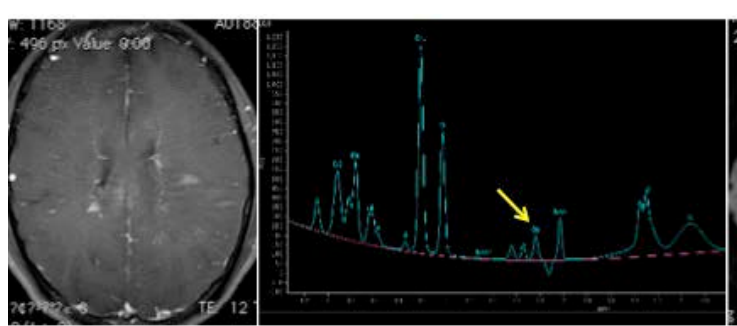

(f) (g)

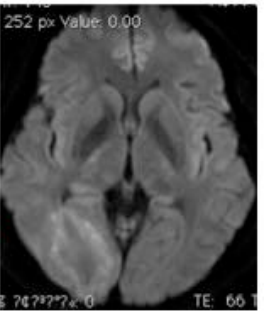

(h)

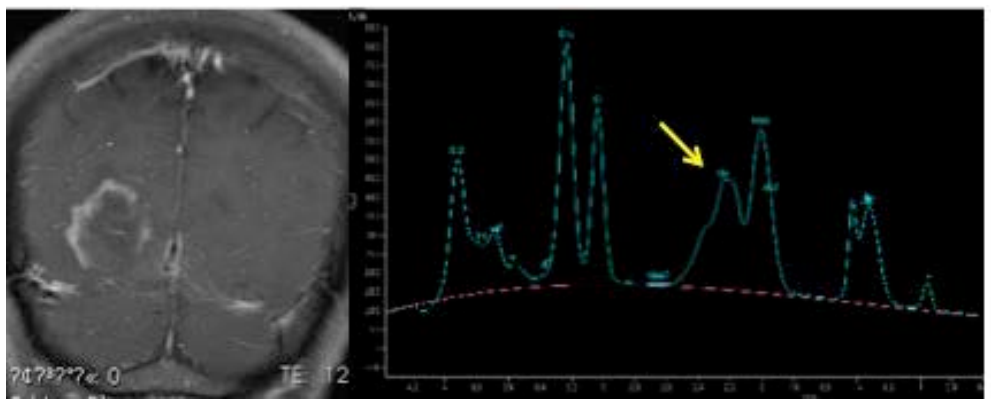

(i)

(j)

Figure 1. Characteristic MRI and MRS findings of 5 patients with TDLs. (a) DWI displayed a peripheral bright rim in Patient 1. (b) Coronal contrast-enhanced T1WI in Patient 1 displayed an open-ring sign, in which the ring of enhancement was open towards the cortex. Mild edema surrounding the lesion was also observed, but the mass effect was mild. (c) DWI displayed a peripheral bright rim in Patient 2. (d) Contrast-enhanced T1WI in Patient 2 showed an open-ring sign, in which the ring of enhancement was open towards the cortex. The lesion was located close to the lateral ventricle, and there was mild edema surrounding the lesion. However, there was almost no mass effect. (e) FLAIR in Patient 3 showed a high signal intensity lesion in the deep white matter of the bilateral parietal lobe, but the mass effect was mild. Inside the lesion, there were a number of vascular flow voids that appeared to intersect the cerebral ventricle. (f) Axial contrast-enhanced T1WI in Patient 3 displayed enhancement of not only the surrounding area of the lesion but also the area around the medullary vein. (g) MRS in Patient 3 displayed an increase in Cho, a decrease in NAA, and the presence of Lac/Lip peaks and $\beta, \gamma$-GLX peaks (arrow) in the core of the lesion. (h) DWI in Patient 5 showed a peripheral high signal, but the signal intensity was heterogeneous. (i) Coronal contrast-enhanced T1WI in Patient 5 displayed an open-ring sign, in which the ring of enhancement was open towards the cortex. Mild edema surrounding the lesion was observed, but the mass effect was very slight. (j) MRS in Patient 5 displayed an increase in the Cho peak and the presence of Lac/Lip peaks at the edge of the lesion, with relatively stable NAA peaks. $\beta$, $\gamma$-GLX peaks (arrow) were also observed.

on autoimmune disorders, removal of the lesion by invasive surgery eliminates the possibility of the recovery of local cerebral function. For the prompt initiation of immunosuppressive treatment, mainly with steroids, there is urgent need for the development of appropriate criteria for preoperative imaging diagnosis of TDLs [1] [2].

Based on our experience with these 5 cases and the evaluation of reported cases, we have identified the following diagnostic imaging characteristics of TDLs:

1) Regarding the localization of the lesion, a large supratentorial lesion with a maximum diameter of $\geq 2 \mathrm{~cm}$ is observed in the region from the paraventricular area of cerebral hemispheres to the brain surface.

2) Most patients have a solitary lesion, but patients can have multifocal lesions.

3) The mass effect on the surrounding brain structure is mild relative to the size of the lesion. 
4) The white matter edema surrounding the lesion is moderate.

5) DWI displays high or bright signal intensity in the surrounding area and low signal intensity at the core. ADC imaging shows high signal intensity at the core.

6) Gadolinium-enhanced imaging displays irregular contrast enhancement of the surrounding area of the lesion, but the enhancement is intense in the deep white matter, with the ring of enhancement being open towards the surface of the brain, showing a so-called open-ring sign.

7) A signal void that appears to involve a vein is observed at the core of the lesion, with enhancement of the surrounding area.

8) Proton MRS displays increases in Cho, Lac, and Lip peaks, with a slight decrease in the NAA peak.

9) Proton MRS shows peaks ( $\beta, \gamma$-GLX peaks) that are not observed in the normal brain, in the range of 2.1 $2.4 \mathrm{ppm}$.

Masdue et al. [3] who reported the open-ring sign for the first time found differences in the intensity of contrast enhancement inassociation with the pathological findings of the removed lesion, which were used to evaluate active inflammatory responses and tissue repair that were predominant in the white matter in demyelinated lesions. Similar pathological findings were also observed in our 3 patients who underwent surgery [1]. Neovascularization associated with tissue repair was observed as a contrast enhancement effect, possibly contributing to the intensity of the edema signal around the lesion [1] [4]. Furthermore, narrowing of the intercellular space due to reactive astrocyte proliferation appeared to have decreased the diffusibility of water molecules [1] [3] [4]. In the center of the demyelinated lesions, an increase in ADC was observed, indicating that widening of the intercellular space associated with the demyelination increased the diffusibility of water molecules. The presence of a vascular signal void that appears to involve a vein at the core of the lesion is important for differentiation between demyelinated lesions and intra-axial tumors [3] [4]. In TDLs, the area around this blood vessel is also enhanced, suggesting that there is marked lymphocyte infiltration in response to demyelination in the perivascular area [1] [3].

Regarding the interesting proton MRS pattern observed, it was considered that the decreased neuronal activity due to demyelination caused a decrease in the NAA peak, and that cell proliferation, through reactive astrocytosis and lymphocyte infiltration, caused an increase in the Cho peak. In addition, the Lac/Lip peaks were considered to be a result of the proliferation of macrophages. Regarding the significance of the $\beta, \gamma$-GLX peaks, which were named "marker peaks" by Grossman et al. [5], Cianfoni et al. [6] reported that when glutamate is released as a result ofrapid demyelination, astrocytes, which essentially control glutamate release, are also severely impaired and become unable to carry out the glutamate-to-glutamine conversion by glutamine synthetase. As a result, due to a significant increase in the glutamate-to-glutamine ratio, the marked glutamate/glutamine peaks emerge. Majós et al. [7] reported that no $\beta, \gamma$-GLX peaks were observed among many patients with intra-axial tumors that we reanalyzed by proton MRS. Therefore, $\beta, \gamma$-GLX peaks can be used to differentiate between TDLs and intra-axial tumors, including glioma, and hence $\beta, \gamma$-GLX peaks appear to be useful marker peaks.

\section{Conclusion}

We analyzed preoperative images obtained from 5 patients who were suspected of having malignant glioma, but were diagnosed with TDLs after detailed examination. As a result, we successfully identified characteristic markers for the diagnosis of TDLs, such as the open-ring sign, a bright rim on DWI, high signal core on ADC imaging, and $\beta, \gamma$-GLX peaks on proton MRS. We expect these findings to be widely diffused, and should enable neurosurgeons to make an accurate diagnosis before surgery, to avoid unnecessary invasive surgery.

\section{Declaration of Interest}

The authors report no conflicts of interest. The authors alone are responsible for the content and writing of the paper.

\section{References}

[1] Akimoto, J., Nakajima, N., Saida, A., Haraoka, J. and Kudo, M. (2006) Monofocal Acute Inflammatory Demyelination Manifesting as Open Ring Sign. Case Report. Neurologia-Medico Chirurgica (Tokyo), 46, 353-357. 
[2] Pittock, S.J., McClelland, R.L., Achenbach, S.J., König, F., Bitsch, A., Brück, W., Lassmann, H., Parisi, J.E., Scheithauer, B.W., Rodriguez, M., Weinshenker, B.G. and Lucchinetti, C.F. (2005) Clinical Course, Pathologic Correlation and Outcome of Biopsy Proven Inflammatory Demyelinating Disease. Journal of Neurology, Neurosurgery Psychiatry, 767, 1693-169. http://dx.doi.org/10.1136/jnnp.2004.060624

[3] Masdue, J.C., Quinto, C., Olivera, C., Tenner, M., Leslie, D. and Visintanier, P. (2000) Open-Ring Imaging Sign: Highly Specific for Atypical Brain Demyelination. Neurology, 54, 1427-1433. http://dx.doi.org/10.1212/WNL.54.7.1427

[4] Lucchinetti, C.F., Gavrilova, R.H., Metz, I., Parisi, J.E., Scheithauer, B.W., Weigand, S., Thomsen, K., Mandrekar, J., Altintas, A., Erickson, B.J., König, F., Giannini, C., Lassmann, H., Linbo, L., Pittock, S.J. and Brück, W. (2008) Clinical and Radiographic Spectrum of Pathologically Confirmed Tumefactive Multiple Sclerosis. Brain, 131, 1759-1775. http://dx.doi.org/10.1093/brain/awn098

[5] Grossman, R.I., Lenkinski, R.E., Ramer, K.N., Gonzalez-Scarano, F. and Cohen, J.A. (1992) MR Proton Spectroscopy in Multiple Sclerosis. American Journal of Neuroradiology, 13, 1535-1543.

[6] Cianfoni, A., Niku, S. and Imbesi, S.G. (2007) Metabolite Findings in Tumefactive Demyelinating Lesions Utilizing Short Echo Time Proton Magnetic Resonance Spectroscopy. American Journal of Neuroradiology, 28, 272-277.

[7] Majós, C., Julia-Sape, M., Alonso, J., Serranllonga, M., Aguilera, C., Acebes, J.J., Arús, C. and Gill, J. (2004) Brain Tumor Classification by Proton MR Spectroscopy: Comparison of Diagnostic Accuracy at Short and Long TE. American Journal of Neuroradiology, 1696-1704. 